\title{
Measurement of Barium lon Displacement Near Surface in a Barium Titanate Nanoparticle by Scanning Transmission Electron Microscopy
}

\author{
Mai Aoki, Yukio Sato*, Ryo Teranishi, Kenji Kaneko \\ Department of Materials Science and Engineering, Kyushu University, Fukuoka 819-0395, Japan
}

*Correspondence to:

Sato $Y$

(iD) http://orcid.org/0000-0002-4537-0692

Tel: +81-92-802-2971

Fax: +81-92-802-2979

E-mail: sato@zaiko.kyushu-u.ac.jp

Received September 20, 2017

Revised October 17, 2017

Accepted October 17, 2017
Barium titanate $\left(\mathrm{BaTiO}_{3}\right)$ nanoparticle is one of the most promising materials for future multi-layer ceramic capacitor and ferroelectric random access memory. It is well known that electrical property of nanoparticles depends on the atomistic structure. Although surface may possibly have an impact on the atomistic structure, reconstructed structure at the surface has not been widely investigated. In the present study, Ba-ion position near surface in $\mathrm{BaTiO}_{3}$ nanoparticle has been quantitatively characterized by scanning transmission electron microscopy. It was found that some Ba ions at the surface were greatly displaced in non-uniform directions.

Key Words: Nanoparticle, $\mathrm{BaTiO}_{3}$, Scanning transmission electron microscopy, Surface, Ferroelectrics

\section{INTRODUCTION}

Barium titanate $\left(\mathrm{BaTiO}_{3}\right.$ : $\left.\mathrm{BTO}\right)$ is a ferroelectric material, which has been used as multilayer ceramic capacitors for long period (Tsurumi, 2007), and is a promising candidate for ferroelectric random access memory (Varghese et al., 2013). Because there has been a strong demand to downsize these devices in recent years, ferroelectric materials of smaller scale such as nanoparticles (NPs), has been intensively studied (Arlt et al., 1985; Frey \& Payne, 1996; Imanaka et al., 2013; Mimura \& Kato, 2014; Tsurumi et al., 2006; Varghese et al., 2013; Zhao et al., 2004). Although it is well known that dielectric and ferroelectric properties of BTO NP primarily depend on the particle sizes (Hoshina et al., 2008; Huan et al., 2014; Imanaka et al., 2013; Smith et al., 2008; Tsurumi et al., 2006; Varghese et al., 2013; Zhao et al., 2004), clear understanding for the size effect has not been obtained. This would be because the situation is complicated by several reasons such as; (i) different studies have reported different values for critical size at which ferroelectric phase becomes unstable at room temperature (Akdogan \& Safari, 2007; Fong et al.,
2004; Ghosez \& Rabe, 2000; Meyer \& Vanderbilt, 2001; Smith et al., 2008; Spanier et al., 2006; Urban et al., 2003), (ii) the phase transition is sensitive to the material's conditions such as strain (Choi et al., 2004), presence of defect (Frey \& Payne, 1996; Hoshina et al., 2006; Yamamoto et al., 2000), and surface charge (Spanier et al., 2006), (iii) crystal structure may not be uniform within a particle (Hoshina et al., 2008), and (iv) BTO NP samples were prepared by some different methods (Bansal et al., 2006; Imanaka et al., 2013; Urban et al., 2003; Varghese et al., 2013).

Since dielectric and ferroelectric properties should be closely related with the crystal structure, crystal structure of BTO NP has been characterized by a variety of methods such as Rietveld analysis (Hoshina et al., 2008; Smith et al., 2008), pair distribution function analysis (Petkov et al., 2006; Smith et al., 2008), X-ray absorption fine structure and X-ray absorption near-edge structure (Frenkel et al., 1999). For example, Smith et al. (2008) reported for BTO NP with the sizes of $25 \mathrm{~nm}, 46 \mathrm{~nm}$, and $70 \mathrm{~nm}$ that paraelectric-to-ferroelectric phase transition was observed for all the samples and the phase transition temperature is almost constant irrespective

@ This is an open-access article distributed under the terms of the Creative Commons Attribution Non-Commercial License (http://creativecommons.org/licenses/by-nc/4.0) which permits unrestricted noncommercial use, distribution, and reproduction in any medium, provided the original work is properly cited.

Copyrights (C) 2018 by Korean Society of Microscopy 
of the particle size, which might be contradictory to the conventional picture that Curie temperature decreases as the particle size decreases (Hoshina et al., 2008; Huan et al., 2014; Imanaka et al., 2013; Smith et al., 2008; Tsurumi et al., 2006; Varghese et al., 2013; Zhao et al., 2004). On the other hand, Hoshina et al. (2008) reported that crystal structure varies within a particle. There is three-layer structure composed of surface (paraelectric), intermediate, and inner (ferroelectric) regions for bigger particle, while the whole particle becomes paraelectric for smaller particle. In analogy to the dielectric/ ferroelectric properties, these examples showed that clear understanding between the particle size and the crystal structure has not been obtained. This also implies that local structural characterization is required in addition to averaged crystal structure characterization.

For the sake of local structural characterization, electron microscopy such as high-resolution transmission electron microscopy or scanning TEM (STEM) is a powerful method, since atomic arrangement can directly be observed. In particular, precision in the atomic position determination has been improved to picometer level in recent years (Borisevich et al., 2012; Jia et al., 2008; Polking et al., 2012; Yadav et al., 2016; Yankovich et al., 2014). Such structural characterization has been reported for example by Polking et al. (2012) with high-resolution TEM and electron holography and by Li et al. (2014) with high-resolution TEM under in-situ heating. Polking et al. (2012) has visualized the polarization distribution within NP and reported that BTO NP with the size below $10 \mathrm{~nm}$ exhibited ferroelectricity at room temperature. On the other hand, Li et al. (2014) has reported that BTO NPs with the sizes ranging from $2.5 \mathrm{~nm}$ to $10 \mathrm{~nm}$ composed of multiple phases and the increment of Curie temperature up to $600^{\circ} \mathrm{C}$. Even though these studies have demonstrated structural distribution in NPs, structure of NP with the size larger than $20 \mathrm{~nm}$ may be needed since such larger NP was not investigated in the previous studies (Li et al., 2014; Polking et al., 2012). Also, both of the studies have suggested that ferroelectric order or crystal phase is affected by the surface. However, the detailed reconstructed structure at the surface has not been clarified. Therefore, in the present study, we report a structural investigation near surface in a BTO NP studied by STEM observation. In particular, displacement of $\mathrm{Ba}$ ion near surface was quantitatively analyzed. Commercially available BTO NP with nominal size of $50 \mathrm{~nm}$ was studied as a specimen.

\section{MATERIALS AND METHODS}

Commercially available BTO NPs (model no. 745952; Sigma-Aldrich, USA; purity: 99.9\%, nominal particle size: $50 \mathrm{~nm}$ ) were investigated in the present study. For comparison, commercially available $\mathrm{SrTiO}_{3}$ single crystals
(Shinkosha, Japan) were also studied. BTO NP powder was homogeneously dispersed in ethanol using ultrasonic cleaner. Subsequently, a small amount of the solution was dropped onto a commercially available TEM grid (NS-C15; Okenshoji, Japan) and the grid was dried in air. On the other hand, a STEM thin foil of the $\mathrm{SrTiO}_{3}$ single crystal was prepared using an FB-2100 focused-ion-beam system (Hitachi HighTechnologies, Japan) with the beam energy of $40 \mathrm{keV}$. Final milling was conducted to remove the damaged layer from the surface by argon-ion-beam milling system (PIPS; Gatan, USA).

STEM observation was carried out using a JEM-ARM200F (JEOL, Japan) equipped with a spherical aberration corrector for the electron probe (Rose, 1994). The observation was carried out under an acceleration voltage of $200 \mathrm{kV}$. TEM images were acquired with an Orius 200D charge coupled device camera (Gatan). Annular dark-field (ADF) STEM images containing $512 \times 512$ pixels were recorded with a detection angle range of 90 and $370 \mathrm{mrad}$ and with a pixel dwell time of $2 \mu \mathrm{s}$. Approximately twenty images were acquired from a single region of interest, and the image series was processed using a non-rigid registration method (Jones et al., 2015) (SmartAlign; HREM Research, Japan) to improve the signal-to-noise ratio. This procedure is useful to reduce the influence of image distortion (Jones et al., 2015). The positions of $\mathrm{Ba}$ and $\mathrm{Ti}$ ions in the image were identified by the peak fitting method. The peak fitting was carried out using the two-dimensional Gaussian function in equation (1), as was done in a previous study (Yankovich et al., 2014),

$Z=Z_{0}+A \exp \left[\left(\frac{-1}{2\left(1-c^{2}\right)}\right)\left\{\left(\frac{x-x_{c}}{w_{x}}\right)^{2}+\left(\frac{y-y_{c}}{w_{y}}\right)^{2}-\left(\frac{2 c\left(x-x_{c}\right)\left(y-y_{c}\right)}{w_{x} w_{y}}\right)\right\}\right]$

where $Z$ and $Z_{0}$ are the total and background intensity, respectively, $A$ is the peak intensity, $x_{\mathrm{c}}$ and $y_{\mathrm{c}}$ are the peak positions in the $x$ and $y$ directions, respectively, $w_{\mathrm{x}}$ and $\mathrm{w}_{y}$ are the width of the Gaussian function in the $x$ and $y$ directions, respectively, and $c$ is a fitting parameter. Differences between the intensities of the experimental image and the Gaussian function $(Z)$ were minimized by the least-squares method by optimizing the parameters of $Z, Z_{0}, A, x_{\mathrm{c}}, y_{\mathrm{c}}, w_{\mathrm{x}}, w_{\mathrm{y}}$ and $c$. The optimization was performed by the Marquart method (Marquardt, 1963) using a script coded using Visual Basic 6 (Microsoft, USA). It should be noticed here that analysis of atomic-column position was conducted both for Ba and Ti ions. However, it was found that the analysis became less accurate in the case of Ti columns near surface for some images, which might be partly due to lower image contrast of Ti column near the surface. Therefore, for clear justification of the conclusion, the present study focuses on the analysis of Ba column positions. 


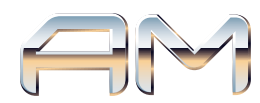

\section{RESULTS AND DISCUSSION}

In prior to the observation of BTO NP, STEM observation of $\mathrm{SrTiO}_{3}$ single crystals was carried out to calibrate the nonsquareness of the scan pixel (Yankovich et al., 2014). Fig. 1A shows the ADF STEM image used for the evaluation. Because the contrast of the ADF STEM image is dependent on the atomic number of constituent ions in the atomic column (Pennycook \& Jesson, 1990), the brightest spot and second brightest spot indicate $\mathrm{Sr}$ and $\mathrm{Ti}$ ions, respectively, whereas the $\mathrm{O}$ ion is not visible. Interatomic spacing between neighboring
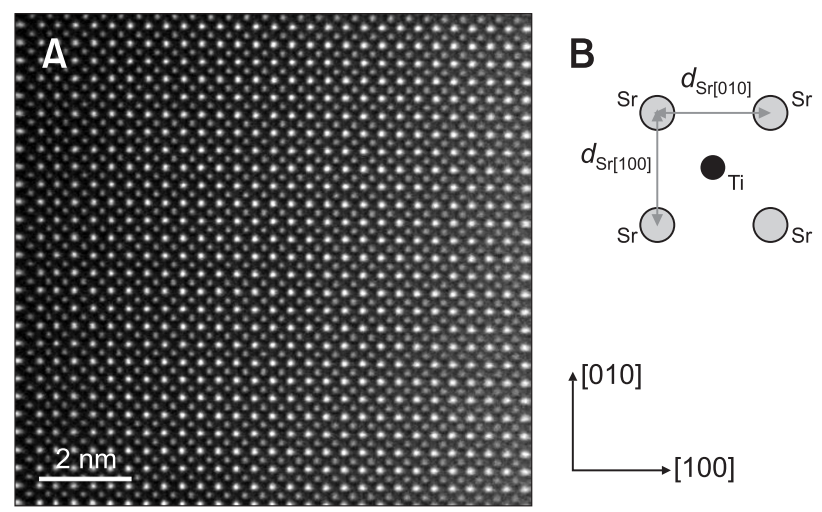

Fig. 1. (A) Annular dark-field scanning transmission electron microscopy image of $\mathrm{SrTiO}_{3}$ single crystal. [100] and [010] direction of STO crystal is shown at the right-bottom. (B) Schematic to explain the definition of $d_{\mathrm{Sr}[100]}$, and $d_{\mathrm{Sr}[010]}$, where Sr ion and Ti ion was indicated by gray and black circle.
Sr ions along the [100] or [010] directions, $d_{\mathrm{Sr}[100]}, d_{\mathrm{Sr}[010]}$, (Fig. 1B) was investigated using peak fitting results, which is similar to the process used by Bals et al. (2006). Because it was found that the left-hand side of the image was considerably distorted, these regions were intentionally excluded from the analysis hereafter. As $\mathrm{SrTiO}_{3}$ has a cubic crystal structure with a lattice constant (a) of $390.5 \mathrm{pm}$ (Mitchell et al., 2000) the ratio between $d_{\mathrm{Sr}[100]}$ and $d_{\mathrm{Sr}[010]}\left(d_{\mathrm{Sr}[100]} / d_{\mathrm{Sr}[010]}\right)$ should ideally be 1.000. On the other hand, it was found in our measurement that the average $d_{\mathrm{Sr}[100]}$ and $d_{\mathrm{Sr}[010]}$ was 17.45 pixels and 17.19 pixels long, resulting in $d_{\mathrm{Sr}[100]} / d_{\mathrm{Sr}[010]}$ of approximately 1.015. Hereafter, the results were calibrated with this ratio. As a result of calibration, the average $d_{\mathrm{Sr}[100]}$ was determined as $390.5 \mathrm{pm}$ with the standard deviation (SD) of $5.8 \mathrm{pm}$ and the average $d_{\mathrm{Sr}[010]}$ was determined as $390.5 \mathrm{pm}$ with the SD of $5.2 \mathrm{pm}$.

Fig. 2A shows a representative TEM image of the BTO NP studied. The particle size was approximately $50 \mathrm{~nm}$, having $\{10 x\}$ and $\{11 x\}$ habit planes on the edge and rounded corners at the atomic scale. It is considered from the image contrast that the particle was composed of multiple domains (Fig. 2B). Presence of multiple domains was also supported by the result that the diffraction spot measured from this particle (Fig. 2C) exhibited splitting (Fig. 2D). A typical ADF STEM image is shown in Fig. 2E, which was taken from the region (I) in Fig. 2B. Image contrast is higher in the grain interior (left top side) and lower near and at the surface (right bottom side), which suggests that the particle is thicker in the grain interior and thinner near and at the surface. For simplicity, the thicker grain-interior region, several unit cells from the surface, and
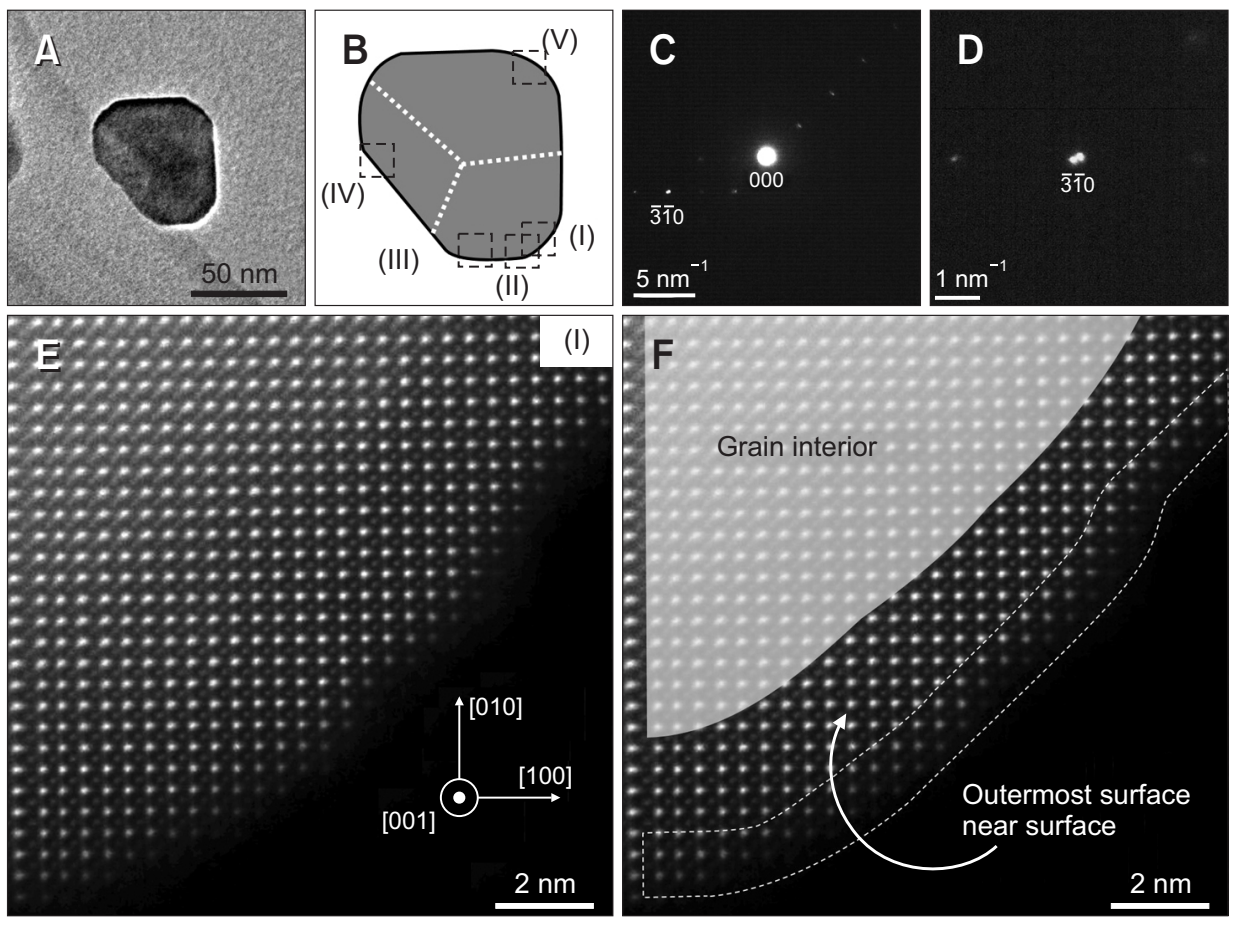

Fig. 2. (A) Transmission electron microscopy (TEM) image of a Barium titanate (BTO) nanoparticle (NP) investigated in the present study. (B) Schematic of the domain structure in the NP, where dotted line indicates domain wall. Black box indicated by (I) (V) are the field of view in the atomic-scale scanning TEM (STEM) image in Fig. 2E and 3A 3D. (C) A diffraction pattern measured from the area including the BTO NP in the image in (A). (D) Magnified $\overline{3} \overline{1} 0$ spot in (C). (E) Atomic scale annular dark-field STEM image taken from the region (I). (F) Definition of "grain interior", "near surface", "outermost surface" as the overlay in the image in (E). 
the outermost layer of the surface will be called the "grain interior", "near surface", and "outermost surface" hereafter, as schematically shown in Fig. 2F.

ADF STEM images (Fig. 2E and Fig. 3) taken from different regions $(\mathrm{I} \sim \mathrm{V})$ as shown in Fig. $2 \mathrm{~B}$ were analyzed to measure the $\mathrm{Ba}$ ion position quantitatively. In particular, $\mathrm{Ba}$ ion displacement from the regular lattice position was determined for the near surface and the outermost surface regions (Fig. 3 ), wherein the reference regular lattice in the near surface and the outermost surface regions was obtained by extending the reference lattice in the grain interior region toward the surface. It was found that some Ba ions were displaced by larger than $20 \mathrm{pm}$ near the surface, although the trends were different for different regions. Ba ions were displaced toward the right-bottom side (outward) in the top half, whereas the displacement in the bottom half was smaller in the region (I) (Fig. 4A). Ba ions were mostly displaced rightward in the outermost surface region in the region (II) (Fig. 4B), while the displacement was smaller and irregular in the region (III) (Fig. 4C). On the other hand, Ba ions in the outermost surface region were mostly displaced rightward (inward) in the region (IV) (Fig. 4D), which was a different trend from the other cases. Finally, in the region (V) (Fig. 4E), Ba ions were displaced upward (outward) in the right half of the outermost surface region, while some ions in the left half of the outermost surface region were displaced leftward nearly along the surface. Thus, some Ba ions were greatly displaced in non-uniform directions. In particular, outward displacement as observed for many $\mathrm{Ba}$ ions in Fig. $4 \mathrm{~A}$ and $\mathrm{B}$ possibly leads to expansion of unit cell near surface, and the influence of the surface would be greater for smaller particle. This may explain by a trend reported by Smith et al. (2008) that unit cell volume increased as the particle size decreased, which was obtained from Rietveld and pair distribution function analysis for the X-ray diffraction measurement. It was also suggested that coherence between the neighboring unit cells was reduced for the smaller NP. Such a reduction of coherence may be induced by Ba-ion displacement toward non-uniform direction (Smith et al., 2008).

In summary, the atomic-scale STEM observation was carried out for near-surface region in a BTO NP with nominal size of $50 \mathrm{~nm}$. Ba-ion position observed in the STEM images was
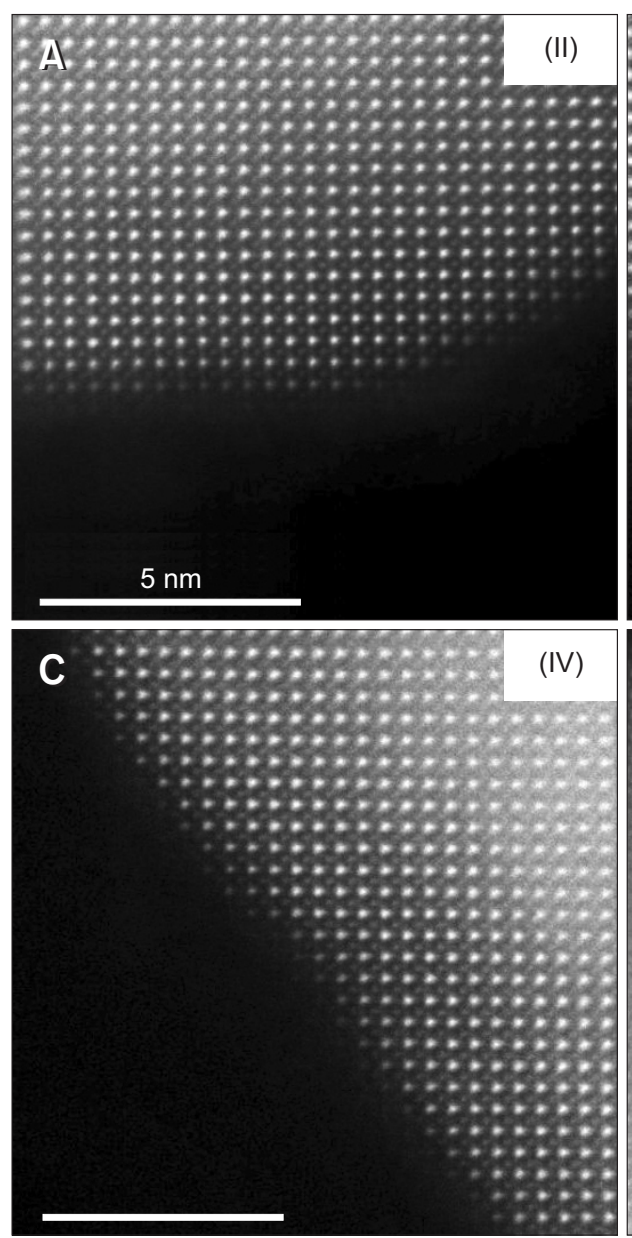

(III)

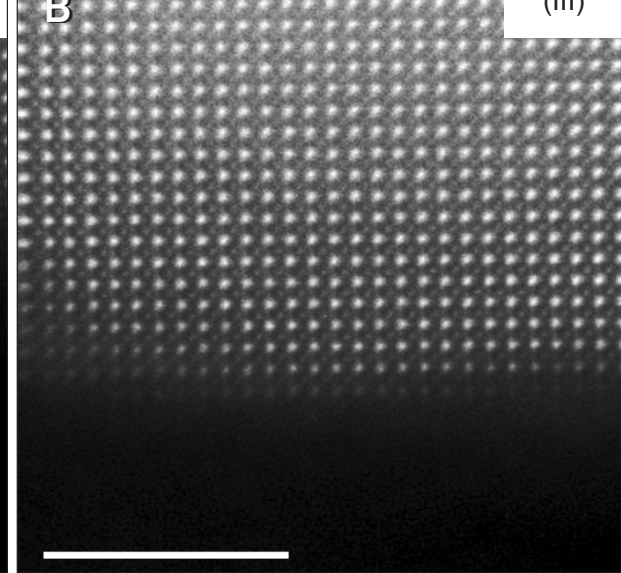

D

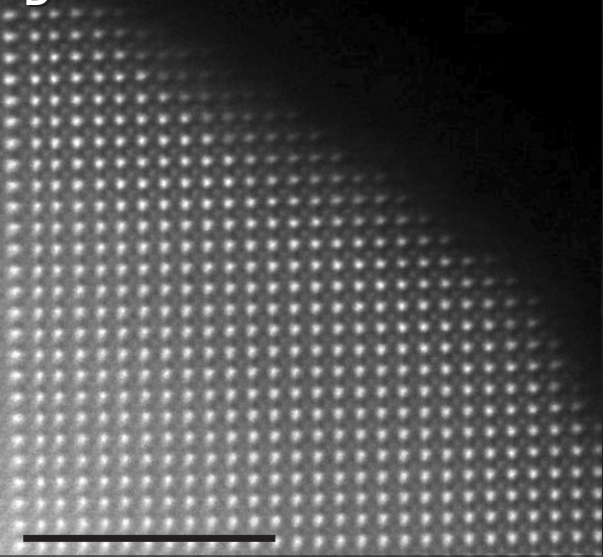

Fig. 3. (A-D) Atomic scale annular darkfield scanning transmission electron microscopy image taken from the region (II) $\sim(\mathrm{V})$. 

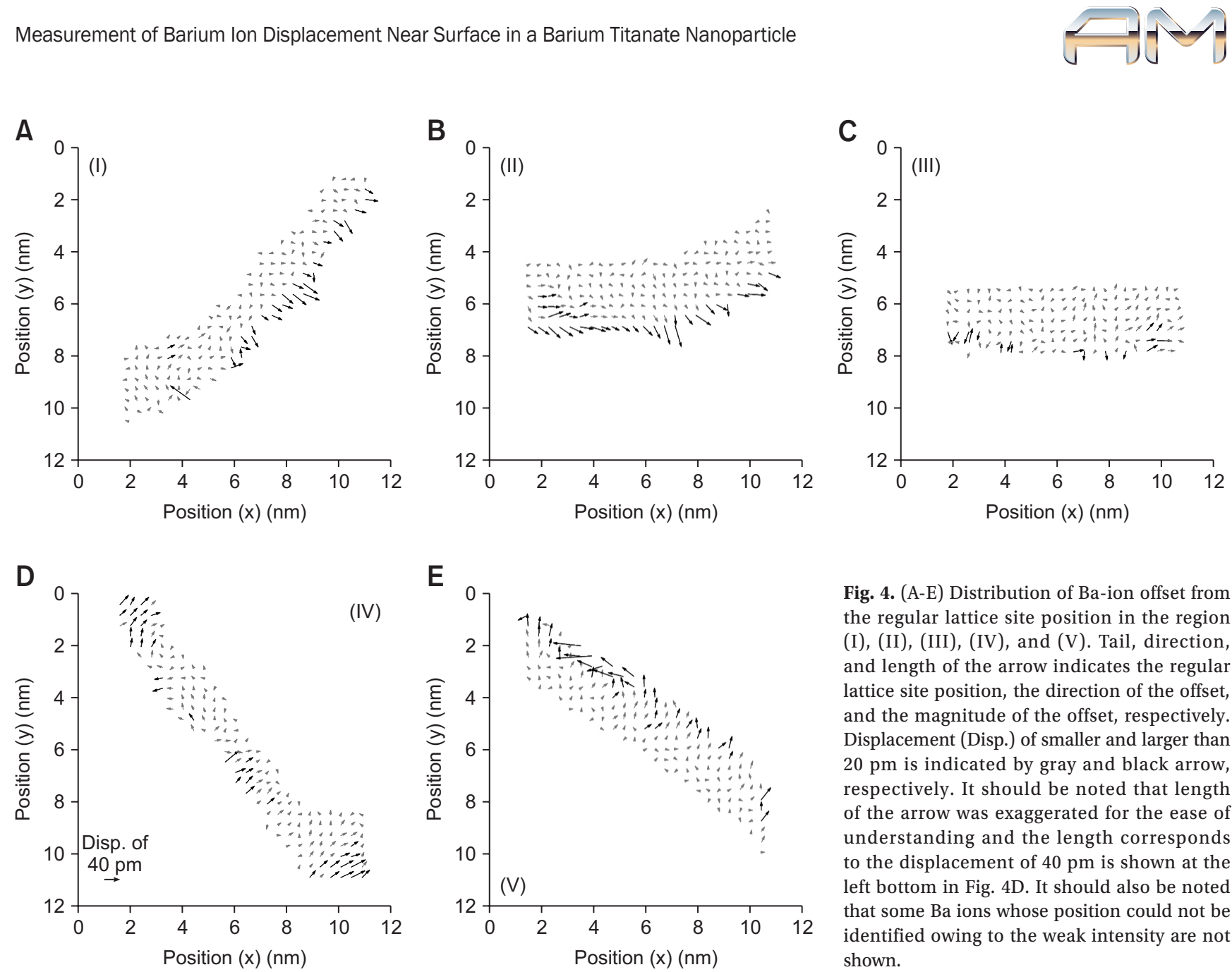

\begin{abstract}
Fig. 4. (A-E) Distribution of Ba-ion offset from the regular lattice site position in the region (I), (II), (III), (IV), and (V). Tail, direction, and length of the arrow indicates the regular lattice site position, the direction of the offset, and the magnitude of the offset, respectively. Displacement (Disp.) of smaller and larger than $20 \mathrm{pm}$ is indicated by gray and black arrow, respectively. It should be noted that length of the arrow was exaggerated for the ease of understanding and the length corresponds to the displacement of $40 \mathrm{pm}$ is shown at the left bottom in Fig. 4D. It should also be noted that some $\mathrm{Ba}$ ions whose position could not be identified owing to the weak intensity are not shown.
\end{abstract}

quantitatively analyzed. It was found that some Ba ions at the outermost surface were greatly displaced in non-uniform directions.

\section{CONFLICT OF INTEREST}

No potential conflict of interest relevant to this article was reported.

\section{ACKNOWLEDGMENTS}

Part of this work was supported by the Grant-in-Aid for Young Scientists (A) (15H05545) and the Grant-in-Aid for challenging Exploratory Research (16K14389) from Japan Society for the Promotion of Science (JSPS). STEM observation was conducted at the Ultramicroscopy Center, Kyushu University.

\title{
REFERENCES
}

Akdogan E K and Safari A J (2007) Thermodynamic theory of intrinsic finite size effects in $\mathrm{PbTiO}_{3}$ nanocrystals. II. Dielectric and piezoelectric properties. J. Appl. Phys. 101, 064114.

Arlt G, Hennings D, and De With G (1985) Dielectric properties of finegrained barium titanate ceramics. J. Appl. Phys. 58, 1619.

Bals S, Van Aert S, Van Tendeloo G, and A' vila-Brande D (2006) Statistical estimation of atomic positions from exit wave reconstruction with a precision in the picometer range. Phys. Rev. Lett. 96, 096106.

Bansal V, Poddar P, Ahmad A, and Sastry M (2006) Room-temperature biosynthesis of ferroelectric barium titanate nanoparticles. J. Am.
Chem. Soc. 128, 11958.

Borisevich A Y, Eliseev E A, Morozovska A N, Cheng C J, Lin J Y, Chu Y H, Kan D, Takeuchi I, Nagarajan V, and Kalinin S V (2012) Atomic-scale evolution of modulated phases at the ferroelectric-antiferroelectric morphotropic phase boundary controlled by flexoelectric interaction. Nat. Commun. 3, 775.

Choi K J, Biegalski M, Li Y L, Sharan A, Schubert J, Uecker R, Reiche P, Chen Y B, Pan X Q, Gopalan V, Chen L Q, Schlom D G, and Eom C B (2004) Enhancement of ferroelectricity in strained $\mathrm{BaTiO}_{3}$ thin films. Science 306, 1005. 
Fong D D, Stephenson G B, Streiffer S K, Eastman J A, Auciello O, Fuoss $\mathrm{P} \mathrm{H}$, and Thompson C (2004) Ferroelectricity in ultrahin perovskite films. Science 304, 1650.

Frenkel A I, Frey M H, and Payne D A (1999) XAFS analysis of particle size effect on local structure in $\mathrm{BaTiO}_{3}$. J. Synchrotron. Radiat. 6, 515.

Frey M H and Payne D A (1996) Grain-size effect on structure and phase transformations for barium titanate. Phys. Rev. B 54, 3158.

Ghosez P and Rabe K M (2000) Microscopic model of ferroelectricity in stress-free $\mathrm{PbTiO}_{3}$ ultrathin films. Appl. Phys. Lett. 76, 2767.

Hoshina T, Kakemoto H, Tsurumi T, Wada S, and Yashima M (2006) Size and temperature induced phase transition behaviors of barium titanate nanoparticles. J. Appl. Phys. 99, 054311.

Hoshina T, Wada S, Kuroiwa Y, and Tsurumi T (2008) Composite structure and size effect of barium titanate nanoparticles. Appl. Phys. Lett. 93, 192914.

Huan Y, Wang X, Fang J, and Li L (2014) Grain size effect on piezoelectric and ferroelectric properties of $\mathrm{BaTiO}_{3}$ ceramics. J. Euro. Ceram. Soc. 34, 1445.

Imanaka Y, Amada H, Kumasaka F, Takahashi N, Yamasaki T, Ohfuchi M, and Kaneta C (2013) Nanoparticulated dense and stress-free ceramic thick film for material integration. Adv. Eng. Mater. 15, 1129.

Jia C L, Mi S B, Urban K, Vreoiu I, Alexe M, and Hesse D (2008) Atomicscale study of electric dipoles near charged and uncharged domain walls in ferroelectric films. Nat. Mater. 7, 57.

Jones L, Yang H, Pennycook T J, Marshall M S J, Aert S V, Browning N D, Castell M R, and Nellist P D (2015) Smart Align-a new tool for robust non-rigid registration of scanning microscope data. Adv. Struc. Chem. Imaging 1, 8.

Li Y, Liao Z, Fang F, Wang X, Li L, and Zhu J (2014) Significant increase of Curie temperature in nano-scale $\mathrm{BaTiO}_{3}$. Appl. Phys. Lett. 105, 182901.

Marquardt D W (1963) An algorithm for least-squares estimation of nonlinear parameters. J. Soc. Indust. Appl. Math. 11, 431.

Meyer B and Vanderbilt D (2001) Ab initio study of $\mathrm{BaTiO}_{3}$ and $\mathrm{PbTiO}_{3}$ surfaces in external electric fields. Phys. Rev. B 63, 205426.

Mimura K and Kato K (2014) Enhanced dielectric properties of $\mathrm{BaTiO}_{3}$ nanocube assembled film in metal-insulator-metal capacitor structure. Appl. Phys. Exp. 7, 061501.

Mitchell R H, Chakhmouradian A R, and Woodward P M (2000) Crystal chemistry of perovskite-type compounds in the tausonite-loparite series, $\left(\mathrm{Sr}_{1-2 x} \mathrm{Na}_{x} \mathrm{La}_{\chi}\right) \mathrm{TiO}_{3}$. Phys. Chem. Minerals 27, 583.

Pennycook S J and Jesson D E (1990) High-resolution incoherent imaging of crystals. Phys. Rev. Lett. 64, 938.

Petkov V, Gateshki M, Niederberger M, and Ren Y (2006) Atomic-scale structure of nanocrystalline $\mathrm{Ba}_{\chi} \mathrm{Sr}_{1-\chi} \mathrm{TiO}_{3}(\chi=1,0.5,0)$ by X-ray diffraction and the atomic pair distribution function technique. Chem. Mater. 18, 814.

Polking M, Han M G, Yourdkhani A, Petkov V, Kisielowski C F, Volkov V V, Zhu Y, Caruntu G, Alivisatos A P, and Ramesh R (2012) Ferroelectric order in individual nanometre-scale crystals. Nat. Mater. 11, 703.

Rose H (1994) Correction of aberrations, a promising means for improving the spatial and energy resolution of energy-filtering electron microscopes. Ultramicroscopy 56, 11.

Smith M B, Page K, Siegrist T, Redmond P L, Walter E C, Seshadri R, Brus L E, and Steigerwald $M L$ (2008) Crystal structure and the paraelectric-to-ferroelectric phase transition of nanoscale $\mathrm{BaTiO}_{3}$. J. Am. Chem. Soc. 130, 6955.

Spanier J E, Kolpak A M, and Urban J J (2006) Ferroelectric phase transition in individual single-crystalline $\mathrm{BaTiO}_{3}$ nanowires. Nano Lett. 6, 735.

Tsurumi T (2007) Non-linear piezoelectric and dielectric behaviors in perovskite ferroelectrics. J. Ceram. Soc. Jpn. 115, 17.

Tsurumi T, Sekine T, Kakemoto H, Hoshina T, Nam S M, Yasuno H, and Wada S (2006) Evaluation and statistical analysis of dielectric permittivity of $\mathrm{BaTiO}_{3}$ powders. J. Am. Ceram. Soc. 89, 1337.

Urban J J, Spanier J E, Ouyang L, Yun W S, and Park H (2003) Singlecrystalline barium titanate nanowires. Adv. Mater. 15, 423.

Varghese J, Whatmore R W, and Holmes J D (2013) Ferroelectric nanoparticles, wires and tubes: synthesis, characterization and applications. J. Mater. Chem. C 1, 2618.

Yadav A K, Nelson C T, Hsu S L, Hong Z, Clarkson J D, Schlep€utz C M, Damodaran A R, Shafer P, Arenholz E, Dedon L R, Chen D, Vishwanath A, Minor A M, Chen L Q, Scott J F, Martin L W, and Ramesh R (2016) Observation of polar vortices in oxide superlattices. Nature (London) 530, 198.

Yamamoto T, Niori H, and Moriwake $\mathrm{H}$ (2000) Particle-size dependence of crystal structure of $\mathrm{BaTiO}_{3}$ powder. Jpn. J. Appl. Phys. 39, 5683.

Yankovich A B, Berkels B, Dahmen W, Binev P, Sanchez S I, Bradley S A, Li A, Szlufarska I, and Voyles P M (2014) Picometre-precision analysis of scanning transmission electron microscopy images of platinum nanocatalysts. Nat. Commun. 5, 4155.

Zhao Z, Buscaglia V, Viviani M, Buscaglia M T, Mitoseriu L, Testino A, Nygren M, Johnsson M, and Nanni P (2004) Grain-size effects on the ferroelectric behavior of dense nanocrystalline $\mathrm{BaTiO}_{3}$ ceramics. Phys. Rev. B 70, 024107. 